\title{
A Computational Model of Chemotaxis-based Cell Aggregation
}

\author{
Manolya Eyiyurekli ${ }^{\text {a,* }}$, Prakash Manley ${ }^{\mathrm{b}}$, Peter I. Lelkes ${ }^{\mathrm{b}}$, David E. Breen ${ }^{\text {a }}$ \\ ${ }^{a}$ Drexel University, Department of Computer Science, Philadelphia, PA 19104 \\ b Drexel University, School of Biomedical Engineering, Science and Health Systems, Philadelphia, PA 19104
}

\begin{abstract}
We present a computational model that successfully captures the cell behaviors that play important roles in 2-D cell aggregation. A virtual cell in our model is designed as an independent, discrete unit with a set of parameters and actions. Each cell is defined by its location, size, rates of chemoattractant emission and response, age, life cycle stage, proliferation rate and number of attached cells. All cells are capable of emitting and sensing a chemoattractant chemical, moving, attaching to other cells, dividing, aging and dying. We validated and fine-tuned our in silico model by comparing simulated 24-hour aggregation experiments with data derived from in vitro experiments using PC12 pheochromocytoma cells. Quantitative comparisons of the aggregate size distributions from the two experiments are produced using the Earth Mover's Distance (EMD) metric. We compared the two size distributions produced after 24 hours of in vitro cell aggregation and the corresponding computer simulated process. Iteratively modifying the model's parameter values and measuring the difference between the in silico and and in vitro results allow us to determine the optimal values that produce simulated aggregation outcomes closely matched to the PC12 experiments. Simulation results demonstrate the ability of the model to recreate large-scale aggregation behaviors seen in live cell experiments.
\end{abstract}

Key words: Chemotaxis, cell aggregation, computational model, computer simulation.

\section{Introduction}

Chemotaxis (CTX) is the phenomenon where cells detect chemical gradients (growth factor, cytokine) and respond to the chemical stimulus by moving either towards (positive CTX) or away (negative CTX) from the source. Multicellular aggregates are formed when individual cells following chemotactic signals encounter and attach to each other. This aggregation often leads to subsequent cellular differentiation and tissue-like assemblies. Understanding the influence of the many components of CTX on overall cell aggregation should lead to a more de-

\footnotetext{
* Corresponding author.

Email addresses: me52@cs.drexel.edu (Manolya Eyiyurekli), prakashmanley@yahoo.co.in (Prakash Manley), pil22@drexel.edu (Peter I. Lelkes), david@cs.drexel.edu (David E. Breen).
}

tailed understanding of the mechanisms involved in tissue assembly and organogenesis, and also facilitate the development of novel technologies for tissue engineering based on controlling or directing these underlying biological processes. Cell-cell aggregation reflects fundamental biological processes occurring during in vivo tissue assembly. Simulating cell aggregates and their assembly/differentiation into functional tissues has implications for the mechanistic understanding of this in vivo process, as well as for establishing models of "in vitro embryology".

We have developed a computational model that is capable of simulating CTX-based cell aggregation in 2-D. Our model incorporates fundamental parameters involved in cell-cell aggregation, such as the ability of individual cells to emit and detect chemoattractants, cell motility, proliferation, aggregation and various stages of a cell's life cycle 
(Eyiyurekli, 2006). Using appropriate approximations/assumptions with efficient and effective algorithms, our model provides a robust and extensible simulation environment. The ultimate goal of our efforts is to produce a powerful, general simulation system that is capable of predicting 3-D cell aggregation/assembly behavior. This capability, and the understanding that it will furnish, will make crucial contributions to our future tissue engineering endeavors by allowing us to study and identify the critical components of cell interaction that most influence aggregation outcomes. We also expect the 3 -D simulation system to be useful for the study of tumor formation in our cancer-related research. While our ultimate goal is to create a 3 -D cell aggregation/assembly simulation system, here we report our initial efforts to develop and validate a 2-D simulation system, which is the first necessary step toward achieving our long-term goal.

In our simulations each cell is defined by a set of physiologically relevant parameters and actions, such as the size and location of the cell, age, life cycle stage, chemoattractant secretion and response rates, diffusion radius, proliferation rate and number of attached cells. Our virtual cells are able to emit chemoattractants, sense the chemoattractant gradient, move in the direction of the gradient, proliferate, adhere to other cells, age and die. We believe that these are the most important features and behaviors needed to simulate chemotaxis-based cell aggregation, and that by including them in our model we are able to represent a wide variety of aggregating cells. In this work we demonstrate that the model is capable of recreating the cell aggregation behavior of at least one type of cell, $\mathrm{PC} 12$ pheochromocytoma cells.

The model's parameters were refined and simulation results were validated by comparisons with in vitro cell-cell aggregation data obtained from an experimental study, in which $\mathrm{PC} 12$ pheochromocytoma cells were allowed to aggregate over a 24hour period. Samples were imaged at the beginning and the end of the experiments and the numbers of individual and aggregated cells were determined. Using the experimentally-derived aggregate size distributions as an outcomes measure, our optimized computational model is capable of reproducing PC12 aggregation behavior. Optimization was accomplished by systematically modifying the parameters of the model, performing 24-hour aggregation simulations, and comparing the resulting in silico aggregate size distributions with the in vitro distributions. By adjusting some of the crucial parameters of our model we are able to address important questions concerning in vitro cell aggregation. The simulation studies showed that parameters such as proliferation rate, quiescent period, chemoattractant release and chemotactic response rates, attachment/detachment probabilities as well as up/downregulation of cell behaviors are key factors that modulate cell aggregation.

\section{Previous Work}

Related work in cell modeling and simulation may be placed in three broad categories, 1) simulation software systems that model the internal workings of a single cell (Tomita et al., 1999), such as cell proliferation and apoptosis, deformation and adhesion, 2) 2-D grid-based models based on cellular automata, ordinary differential equations, or a combination of the two (Graner and Glazier, 1992), and 3) a hybrid representation of cells as discrete units that move in a continuous space (Fleischer and Barr, 1994). The grid-based approach has been used to simulate cell sorting, the morphogenesis of numerous cell-level biological structures, and tumor development. It has recently been extended to 3-D (Cickovski et al., 2005). The hybrid approach has been used to simulate morphogenesis, organism motility, and chemotaxis. It also has been extended to 3-D to create simple geometric structures (Fleischer, 1995), and has found limited use in modeling more complex biological processes and systems (Chaturvedi et al., 2005).

There are several single cell-based computational models that simulate dynamic behavior of single cells under the effect of intra-cellular molecular interactions. These models analyze changes in expression levels of proteins and other chemical compounds within a cell (Tomita et al., 1999) to predict cellular behavior such as growth, signaling, proliferation, differentiation and death (Christopher et al., 2004; Slepchenko et al., 2003). These approaches have been used to simulate several intracellular processes such as energy production and phospholipid synthesis (Tomita et al., 1999), and to simulate intracellular calcium dynamics and nucleocytoplasmic transport (Slepchenko et al., 2003) as well as intercellular processes like bacterial chemotaxis (Emonet et al., 2005). A more detailed approach to modeling and simulation of gene-gene, geneprotein and protein-protein interactions focuses 
on sporulation, metabolismic activity, signaling, sensing and apoptosis (Bio-SPICE, 2008), while a more general approach to cell simulation provides a modular meta-algorithm that can incorporate any time-driven simulation algorithm (Takahashi et al., 2004). An implementation of this meta-algorithm can be found in Tomita et al. (1999).

2D and 3D grid based models focus on intercellular processes such as signaling, motion and attachment to an extracellular matrix. Some of these models also combine the internal workings of individual cells with external processes (Izaguirre et al., 2004). These models treat cells as particles collectively moving on a rectilinear grid.

The Cellular Potts Model (CPM) (Graner and Glazier, 1992), a lattice-based stochastic framework, has been used to model adhesion driven motion created by varying surface energies of different kinds of cells in order to simulate cell sorting. CPM has been used as a part of other models to simulate the rearrangement of cells during morphogenesis (Chaturvedi et al., 2005; Glazier and Graner, 1993) and to model avascular tumor growth (Jiang et al., 2005). A discussion of how cell-centered simulations like CPM can help to explain aspects of developmental biology can be found in Merks and Glazier (2005). Some models in this category (Hogeweg, 2000, 2002; Tozeren et al., 2005) use cellular automata (Ilachinski, 2001; Wolfram, 1984) to model cell motility and differentiation in order to simulate tumor growth (Dormann and Deutsch, 2002; Patel et al., 2001) and embryogenesis (Longo et al., 2004).

Other models that use a grid-based approach, combined with environmental forces, simulate the deformation and aggregation of red blood cells in a blood flow (Bagchi et al., 2005) and the formation of fruiting bodies (Sozinova et al., 2006). Monte-Carlo models, non-deterministic models that simulate a solution by random assignments to uncertain model variables, using only Brownian Motion (Song et al., 2004) and a combination of Brownian Motion and cellular automata (Ascencio et al., 2001) have been developed to simulate tumorigenesis.

The third approach utilizes hybrid models that contain both discrete and continuous components. These models define cells as independent, discrete units that can move in response to continuous forces and influences. This approach has found use in simulating multicellular pattern formation (Fleischer and Barr, 1994), studying the chemotactic motility of individual cells (Jabbarzadeh and Abrams, 2005; Palsson and Othmer, 2000) and the computational modeling of cell adhesion (N'Dri et al., 2003).

Some models integrate two or more of these approaches in one simulation system. COMPUCELL2D (Izaguirre et al., 2004) and 3D (Cickovski et al., 2005) have been developed with both CPM and diffusion-based continuum models, and are able to simulate cell-cell signaling and cells moving in response to adhesion forces. Newman et al. (2008) have used a hybrid approach for multiscale modeling of vertebrate limb development.

There has been some use of Genetic Algorithms (GAs) (Goldberg, 1989) with both grid based and hybrid computational models for simulating cell differentiation (Hogeweg, 2000, 2002), morphogenesis (Kumar, 2004), organogenesis (Chaturvedi et al., 2005) and embryogenesis (Longo et al., 2004).

Our hybrid model simulates chemotaxis, where chemical-based cell-cell signaling is the main source of interaction. A similar use of cell-cell signaling can be found in Jabbarzadeh and Abrams (2005) and Fleischer and Barr (1994). While our model contains many of the elements from previous efforts (Cickovski et al., 2005; Fleischer, 1995, 1996; Fleischer and Barr, 1994; Izaguirre et al., 2004; Jabbarzadeh and Abrams, 2005), e.g. discrete cells that move, divide and attach, it also considers the affect of apoptosis on aggregation outcomes, a feature not found in other models. More importantly though, our work "closes the loop" on the study of cell aggregation by quantitatively comparing the results from a simulated cell environment with cell aggregation outcomes observed in actual experiments. Iteratively coupling simulations and in vitro experiments has allowed us to validate and fine-tune the model, as well as identify some of the critical components of cell interaction that affect large-scale cell aggregation behavior. None of the previous work in this area has performed this type of quantitative comparison and validation with experimental results derived from actual cell aggregations. Having our model faithfully reproduce the outcomes from in vitro experiments demonstrates the beginnings of a predictive cell simulation capability.

\section{Methodology}

\subsection{Theoretical Model}

We present a 2-D computational model, and associated simulation system, of chemotaxis-based cell aggregation that captures the large-scale character- 
istics of PC12 cell aggregation behavior. Our model consists of individual cells existing in an environment that emit and respond to chemoattractants. For the sake of simplicity we assume that there is only one chemoattractant. Each cell detects the state of the chemoattractant-containing environment, and responds to the environment based on a pre-defined "program" and its own internal state. The model is hybrid in nature because it includes both discrete and continuous components. The cells are discrete processing units that are located on a grid, exist in discrete states (e.g. active or dying) and perform discrete tasks (e.g. divide and attach), but they also contain and are affected by continuous quantities (e.g. chemoattractant concentrations, gradients, age and velocities).

\subsubsection{Cell Life Cycle}

During its lifetime a cell passes through different stages (Lodish et al., 1995). The life cycle of a cell is depicted in Figure 1. Every cell is in the Active stage at the beginning of a simulation run. New cells are created via cell division. A dividing cell creates two daughter cells which may separate and move away from each other during the Dividing phase. They might also stay together with a certain probability to create larger aggregates. After a cell is created by cell division it enters a Quiescent phase where its biological machinery is not fully functional. These cells slowly increase their chemoattractant production and respond to chemoattractants at an increasing rate in this stage. The cells either follow a gradient or take a random step with a decreasing probability during quiescence. A quiescent cell can attach to other cells upon collision. Following this period a cell becomes most active, and is able to emit a chemoattractant, form aggregates and/or divide to create new cells. If a cell fails to either divide or attach during the active period the cell moves into a programmed cell death (apoptosis) Dying phase. During apoptosis a cell begins to shut down, resulting in a decrease in the secretion rate of chemicals and a lowered response to chemicals in the environment. Cells undergoing apoptosis are still able to move, encounter other cells and attach, but will not divide to create new cells. Once apoptosis begins the cell dies within a certain time period, and is no longer included in the simulation.

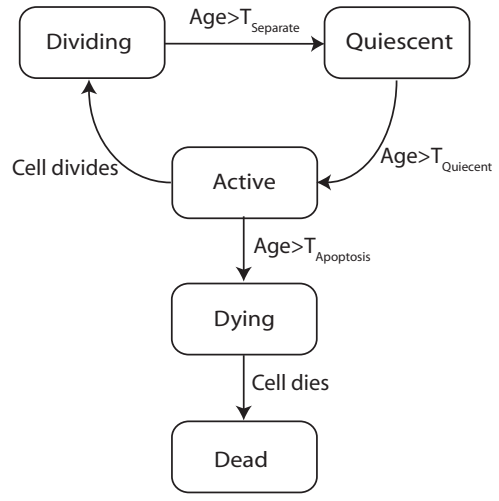

Fig. 1. Life cycle of a cell: Cells exist in five main stages - Dividing, Quiescent(after division), Active, Dying (apoptosis) and Dead.

\subsubsection{Chemoattractant Emission}

All virtual cells live on a $3225 \times 2792 \mu \mathrm{m}$ grid representing a $2-\mathrm{D}$ cross-section of a small portion of a Petri dish. Each cell has a radius of $6 \mu \mathrm{m}$. Chemoattractants are secreted from the cell's surface symmetrically and diffuse within a fixed radius of influence. The concentration of chemoattractant initially secreted by a single cell at its surface is $C_{0}$ molecules $/ \mu m^{2}$. The concentration is up/downregulated by a number of factors, including age, cell cycle state and number of attached cells. Diffusion of the chemoattractant decreases the chemical concentration in the region around the cell as a function of distance from the cell's surface. We assume that a cell's emission of chemoattractants maintains a constant chemical concentration at the cell's surface, creating a static chemical concentration field around each cell. Given this assumption, the chemoattractant concentration within the field drops off as $1 / r$, where $r$ is the distance from the cell surface (Crank, 1975).

Chemoattractant emission from a cell's surface is a function of its state and is controlled by two constants $k_{1}$ and $k_{2}$ (Figures 2 and 4$)$.

$$
\begin{gathered}
k_{1}= \begin{cases}0 & 0 h \leq \text { Age }<T_{\text {Awake }} \\
F_{1}(\text { Age }) & T_{\text {Awake }} \leq \text { Age }<T_{\text {Quiescent }} \\
1 & T_{\text {Quiescent }} \leq \text { Age }<T_{\text {Apoptosis }} \\
F_{2}(\text { Age }) & T_{\text {Apoptosis }} \leq \text { Age }<T_{\text {Death }}\end{cases} \\
F_{1}(T)=0.5-0.5 * \\
\cos \left(\pi *\left(T-T_{\text {Awake }}\right) /\left(T_{\text {Quiescent }}-T_{\text {Awake }}\right)\right) \\
F_{2}(T)=0.5+0.5 * \\
\cos \left(\pi *\left(T-T_{\text {Apoptosis }}\right) /\left(T_{\text {Death }}-T_{\text {Apoptosis }}\right)\right)
\end{gathered}
$$




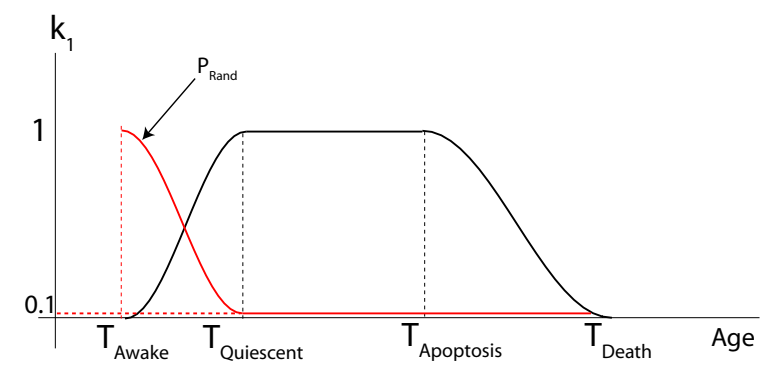

Fig. 2. $k_{1}$ : Change in chemoattractant emission and response as a function of age. $P_{\text {Rand }}$ : Probability that a quiescent single cell will move randomly.

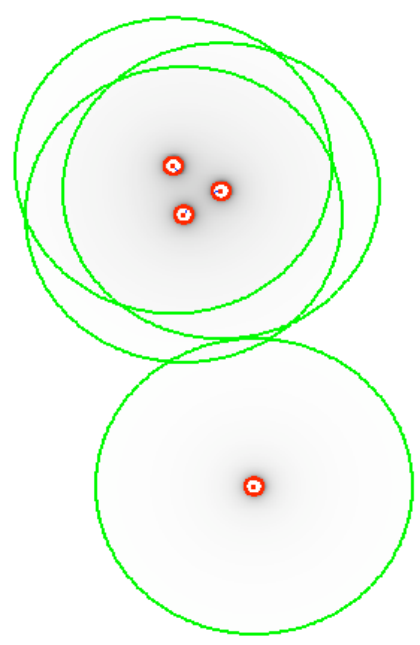

Fig. 3. Four cells and their fields of influence. A cell senses the chemicals emitted from other cells when it enters their influence fields. The extent of the field is defined by a chemical concentration threshold. Three cells on the top are affected by each other's chemoattractant. The fourth cell is outside their influence fields, and therefore is unaffected.

The function used to calculate the chemoattractant concentration around a cell is

$$
C(r)=A+\frac{k_{1} * k_{2} * C_{0}}{1+r} .
$$

$k_{1}$ increases/decreases a cell's chemoattractant output if it is quiescent or has begun apoptosis (Kobayashi et al., 2003) (See Section 3.1.6), and $k_{2}$ regulates chemoattractant emission based on the number of neighboring cells attached to the current cell.

We assume that once the chemoattractant concentration falls below a certain value, cells in the environment will no longer respond to the chemoat- tractant. This assumption defines a circular field around each cell with a radius of $R_{\text {Max }}$. Any cell within a distance less than $R_{\text {Max }}$ to another cell is influenced by the gradient of the chemoattractant emitted by the other cell. A cell that is further away than $R_{\text {Max }}$ from an emitting cell does not detect its chemoattractant and the detecting cell's motion is not affected by the emitting cell. For example, the three cells on the top in Figure 3 are within each other's field of influence, and move toward each other. The fourth cell is not affected by the other three and moves randomly.

$A$ is a constant that allows us to set the chemoattractant concentration at $R_{M a x}$. If we assume that $C(r)$ is some percentage of the initial chemical concentration on the cell surface (percent $* C_{0}$ ) when $r=R_{\text {Max }}$, we can solve for $A$ (assuming that $k_{1}=$ $\left.k_{2}=1\right)$,

$$
A=C_{0} * \text { percent }-\frac{C_{0}}{1+R_{\text {Max }}} .
$$

Note that with this definition of $A$ that $C\left(R_{\operatorname{Max}}\right)=$ $C_{0} *$ percent. This easily allows us to define the chemoattractant concentration at $R_{M a x}$ as a percentage of the concentration at the cell surface. We solve this equation for a single cell where $R_{\operatorname{Max}}=$ $R_{0}$ and percent is 0.01 (1\%). The values for $C_{0}$ and $R_{0}$ for single cells can be obtained from the literature (see Table 1 ). Setting $C_{0}$ and $R_{0}$ to these initial values and $k_{1}=k_{2}=1.0$ in Equation 3 produces the value of $\mathrm{A}$ for a single cell. Once the value for $A$ is calculated for the initial conditions of a single cell it remains constant throughout the simulation. With A held constant, $R_{\text {Max }}$ is calculated for each cell at each time step. As $C(r)$ changes with the changing values of $k_{1}$ and $k_{2}$, a new $R_{M a x}$ must be calculated to determine the maximum size of the detectable chemical field, i.e. the distance where the chemical concentration is $C_{0} *$ percent. Incorporating Equation 3 into Equation 2, setting $C(r)$ equal to $C_{0} *$ percent, and solving for $R_{M a x}$ gives

$$
R_{\text {Max }}=\frac{k_{1} * k_{2} * C_{0}}{C_{0} * \text { percent }-A}-1 .
$$

The number of neighbors attached to a cell affects its secretion process. As a cell acquires more neighbors the chemoattractant emission is up/downregulated. There are four possible scenarios for this regulation:

(i) a cell's response is down-regulated as more cells are attached,

(ii) the number of attached cells does not affect chemoattractant emission, 
Table 1

Constant parameter values used in the computational model

\begin{tabular}{|l||l||l|}
\hline Parameter & Description & Value \\
\hline$\Delta t$ & Time step for simulation state update & $60 \mathrm{~s}$ \\
\hline$d$ & Grid discretization & $1 \mu \mathrm{m}$ \\
\hline Dim & Grid dimension & $3225 \times 2792 \mu \mathrm{m}^{2}$ \\
\hline$C_{0}$ & Chemoattractant concentration at cell surface & 90 molecules $/ \mu \mathrm{m}^{2}$ (Savinell et al., 1989) \\
\hline$R_{0}$ & Initial radius of influence & $100 \mu \mathrm{m}$ (Serini et al., 2003) \\
\hline$r_{c}$ & Cell radius & $6 \mu \mathrm{m}$ (Watanabe et al., 1983) \\
\hline$T_{\text {Apoptosis }}$ & Start time of apoptosis & $18 \mathrm{~h}$ (Hirata et al., 1998) \\
\hline$T_{\text {Death }}$ & End time of apoptosis. All single cells die. & $24 \mathrm{~h}$ (Hirata et al., 1998) \\
\hline$N_{0}$ & Initial cell count & 885 \\
\hline
\end{tabular}

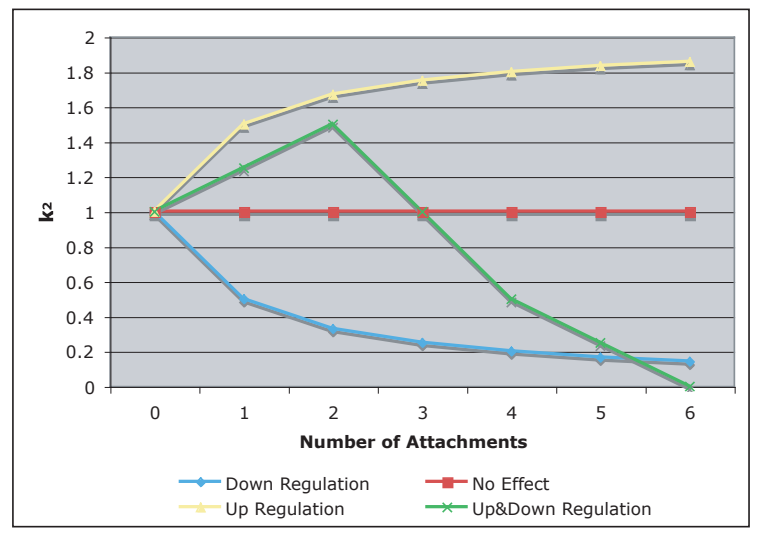

Fig. 4. $k_{2}$ : Chemoattractant emission scale factor. The emission of chemoattractant is up/down-regulated as a function of the number of attached cells. Four possible scenarios are presented. Red: no up/down-regulation. Yellow: up-regulation. Blue; down-regulation. Green: up-regulation, followed by down-regulation.

(iii) a cell's response is up-regulated as more cells are attached, and

(iv) a cell's response is first up-regulated for a certain number of attached cells and then downregulated as even more cells are attached.

The first scenario implements a form of contact inhibition of motility and migration (Huttenlocher et al., 1998). The third scenario implements the opposite response of contact stimulation of cell migration (Thomas and Yamada, 1992) and protein synthesis (Wall et al., 1998). The fourth scenario is one that we postulated could be caused initially by contact stimulation, but as more cells attach they interfere with diffusion of the chemoattractant.
These behaviors are modeled with the scale factor $k_{2}$ in Equation 2. The values of $k_{2}$ used in our simulations are presented in Figure 4. After running and evaluating several simulations, optimal simulation/modeling of the experimental data was obtained when utilizing the first scenario. Here, the chemoattractant emission is down-regulated as the number of attached cells increases. Initially $k_{2}$ is 1 , and then it steadily decreases as $1 /(n+1)$, where $\mathrm{n}$ is the number of attached cells.

\subsubsection{Chemoattractant Sensing}

Chemical signals from other cells in the vicinity are sensed via cognate receptors located symmetrically on the cell's surface. The chemoattractant concentrations sensed at these receptors are used for the gradient calculation. We assume that each cell has eight receptors evenly distributed over its surface, a distance $r_{c}$ from the cell center. The placement and numbering of the receptors, within the local coordinate system of the cell, begins at 45 degrees in the upper right of the cell and proceeds clockwise (see Figure 5). If $\Lambda_{i}$ refers to the chemical concentration detected at receptor $r_{i}$ of a single cell, the gradient is calculated as: 


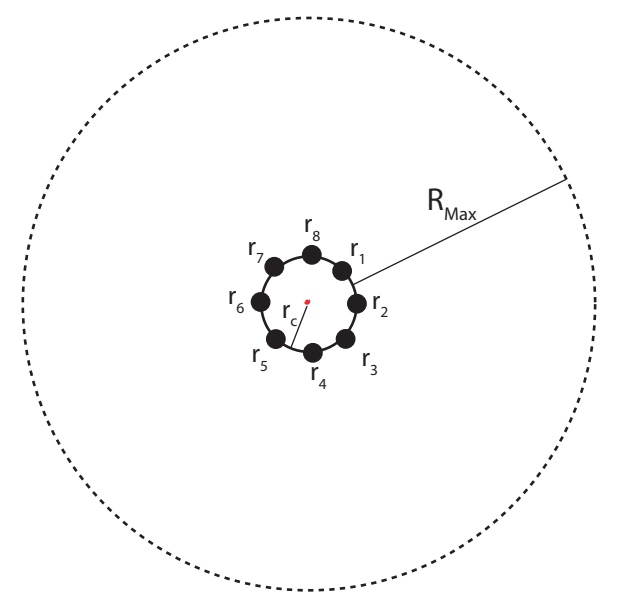

Fig. 5. A virtual cell has eight receptors on its surface $r_{1} . . r_{8}$. The chemoattractant emitted from the cell surface diffuses within a radius of $R_{\operatorname{Max}}$. The chemoattractant falls below a detectable level outside of $R_{M a x}$.

$$
\begin{array}{r}
x=\frac{\Lambda_{2}-\Lambda_{6}+\frac{\Lambda_{1}-\Lambda_{5}-\Lambda_{7}+\Lambda_{3}}{\sqrt{2}}}{2 * r_{c}} \\
y=\frac{\Lambda_{8}-\Lambda_{4}+\frac{\Lambda_{7}+\Lambda_{1}-\Lambda_{5}-\Lambda_{3}}{\sqrt{2}}}{2 * r_{c}} \\
\nabla C=(x, y) \\
|\nabla C|=\sqrt{x^{2}+y^{2}} \\
\Theta(\nabla C)=\operatorname{atan}(y / x)
\end{array}
$$

\subsubsection{Cell Motility and Attachment}

The chemoattractant gradient is used to determine the direction and magnitude of an Active cell's velocity. An Active cell's motion is usually in the direction of this local gradient, however $1 \%$ of the time each cell will take a random step of length 1-3 $\mu \mathrm{m}$, producing a velocity equal to $60-180 \mu \mathrm{m} /$ hour (Farrell et al., 1990) instead. We assume that cells travel at a terminal velocity through the viscous fluid environment, therefore a virtual cell's velocity is directly proportional to the chemical gradient $(\nabla C)$ detected at the cell's surface. When a cell moves in the direction of the chemical gradient, its velocity is calculated as

$$
\text { Velocity }=\lambda * k_{1} * \nabla C,
$$

(Song et al., 2006) where $\lambda$ is a constant that determines the magnitude of a cell's response to the gradient and $k_{1}$ increases/decreases the response during quiescence/apoptosis. At each simulation time step $(\Delta t)$ the displacement of the cell/aggregate is

$$
\Delta \mathbf{x}=\text { Velocity } * \Delta t .
$$

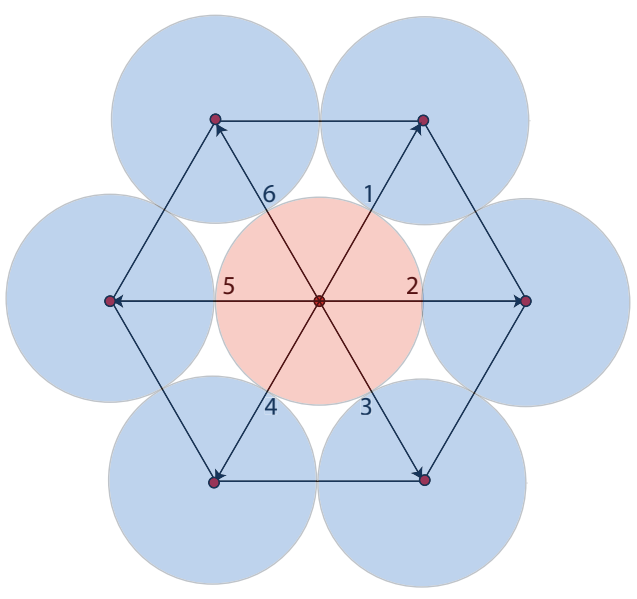

Fig. 6. The cell at the center can move in and be attached at one of the six directions defined by the hexagonal grid. The black dots represent the cell centers and large circles represent the cells.

We postulate that when two cells collide they attach to each other. Since all cells have the same radius and move within a hexagonal grid, there are six fixed cell-cell binding sites on a boundary of a $2 \mathrm{D}$ cell (See Figure 6). We assume that once an attachment is made it cannot be broken; thus creating aggregates of increasing size. Once cells attach to each other and form an aggregate a single velocity is calculated for the aggregate by averaging the gradients calculated for each of its individual cells. The aggregate then moves in the direction of the average gradient with a velocity equal to

$$
(\lambda / M) * \nabla C_{\text {avg }},
$$

where $M$ is the mass of the aggregate, i.e. the number of cells in the aggregate.

\subsubsection{Cell Proliferation}

A cell in the Active phase can divide to create two new cells. In our model cell division occurs by randomly adding a new cell to one of the open six binding sites on the dividing cell's surface. The probability of proliferation is affected by the number of attached cells. A cell becomes less productive as the number of its attachments grows, a form of contact inhibition (Castor, 1972; Schutz and Mora, 1968). We assume that the decreasing probability of cell division can be defined as $e^{-n}$, where $n$ is the number of attached neighboring cells. As seen in Figure 7, a cell with six attachments is considered fully surrounded, and can no longer divide. Thus, single cells and cells on the outer layer of an aggregate have higher rates of proliferation compared to cells found 


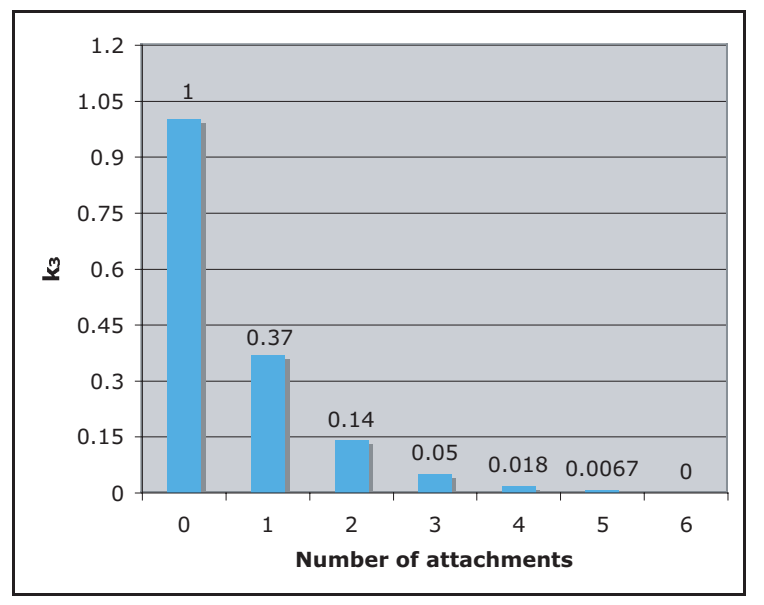

Fig. 7. $k_{3}$ : Change in probability of proliferation as a function of the number of attached cells. These values approximate $e^{-n}$.

inside of an aggregate and surrounded by other cells. The probability of proliferation during a simulation time step is

$$
P_{\text {Div }}=k_{3} * P_{D i v}^{0}
$$

where $P_{D i v}^{0}$ (see Table 3 ) is the probability that a single cell will divide during a simulation time step. $k_{3}$ lowers the probability of proliferation as the number of attachments increases. $P_{D i v}^{0}$ is set during initialization and stays constant throughout the simulation for every cell.

Once proliferation (cell division) occurs, the daughter cells enter the Dividing phase, and may not necessarily stay attached. The probability that two daughter cells will separate after division $\left(P_{S e p}\right.$, see Table 3 ) is set during initialization and stays constant throughout the simulation for every cell. The Dividing period lasts $T_{\text {Awake }}$ minutes, and upon completion all newly-proliferated daughter cells enter an inactive (Quiescent) state in their life cycle. $T_{\text {Awake }}$ is defined to be half of $T_{\text {Quiescent }}$, the time when the Quiescent period ends. In this phase the cells are not fully functional in that they do not initially emit chemoattractant or respond to chemoattractant gradients. The cells gradually activate these functionalities as they age. They cannot proliferate, however, they can attach to other cells. They move randomly in the environment with a decreasing probability $P_{\text {Rand }}$ (see Figure 2) and follow the chemoattractant gradient with probability $1-P_{\text {Rand }}$. Single inactive cells move randomly with a velocity of $60-180 \mu \mathrm{m} /$ hour $(1$ to $3 \mu \mathrm{m}$ per 1 minute time step). Inactive cells attached to an aggregate move with the aggregate, with their

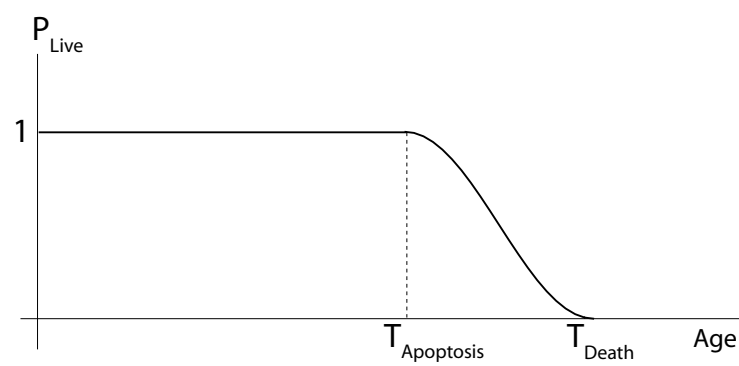

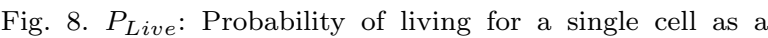
function of age. The probability goes to zero after $T_{\text {Apoptosis }}$ if the cell is unattached. All apoptotic cells die by $T_{\text {Death }}$.

velocity being determined by the velocity of the aggregate. Once the Quiescent period ends the cell fully emits and responds to chemoattractants, and once again can divide.

\subsubsection{Cell Death}

Cells are more likely to survive, i.e. stay alive, when they are attached to other cells. If a cell is still unattached after $T_{\text {Apoptosis }}$, it begins apoptosis (in this case through a mechanism termed anoikis, cell death caused by "homelessness" (Frisch and Francis, 1994)). During apoptosis a cell's chemoattractant emission and response rates decrease over time, and proliferation stops completely. The affect of apoptosis on a cell is implemented with the scale factor $k_{1}$. (see Figure 2 and Equation 1). In the Dying phase $k_{1}$, because of its inclusion in Equations 2 and 6, progressively reduces the amount of chemoattractant emitted by the cell and slows the cell's response to chemical gradients (Kobayashi et al., 2003). The actual time of cell death is also controlled by the parameter $P_{\text {Live }}$. $P_{\text {Live }}$ can be interpreted as the probability of living for a single cell as a function of age. The probability is 1.0 up to $T_{\text {Apoptosis }}$, after this time an unattached cell has an increasing probability of dying, up to $T_{\text {Death }}$ (24 hours in our simulation), when all remaining apoptotic cells die (See Figure 8). Once a cell dies it is removed from the simulation. It is assumed that the cell breaks up and it is not counted when collecting aggregation distribution information. A cell can still attach to other cells during the Dying phase. However once beginning apoptosis, forming an attachment does not prevent a cell from dying by $T_{\text {Death }}$. 


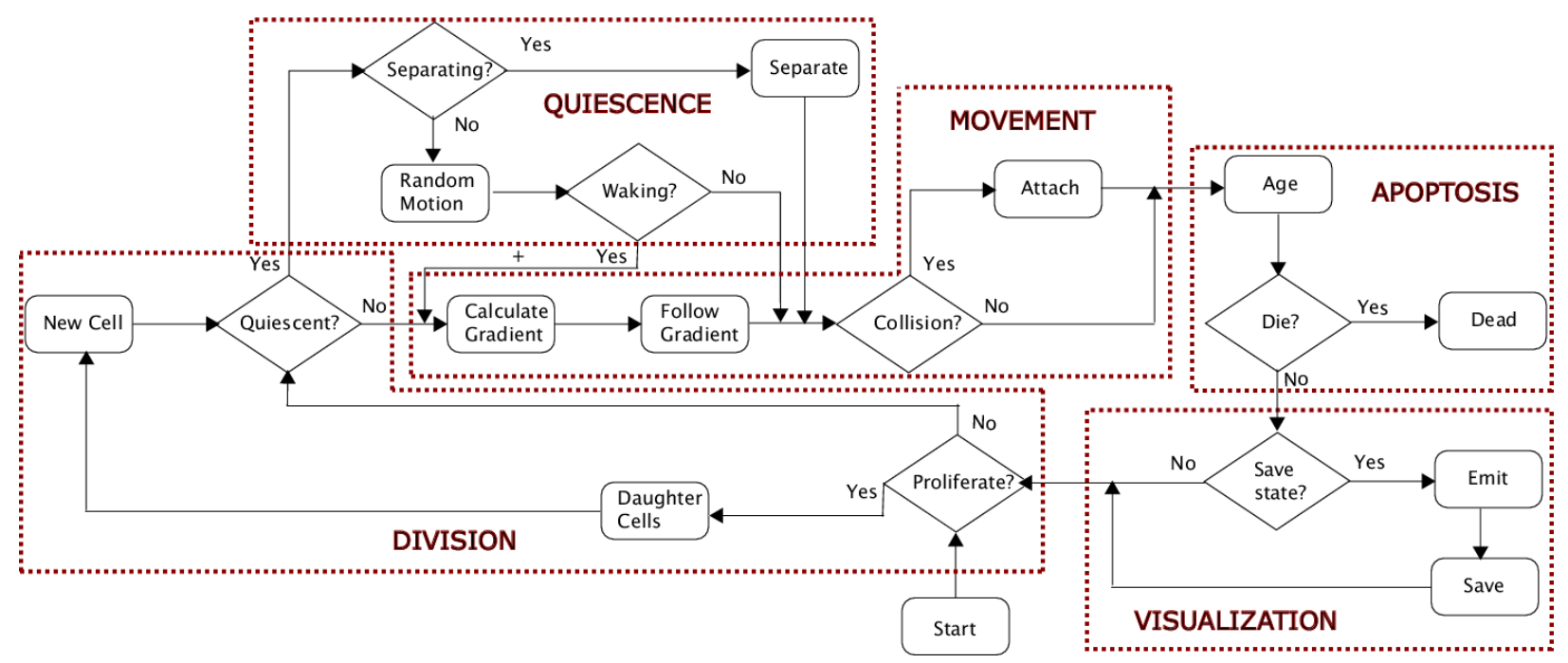

Fig. 9. Computational flow of cell aggregation simulation per time step per cell.

Table 2

Actions taken by a virtual cell.

\begin{tabular}{|c|c|}
\hline Function & Description \\
\hline Emit() & $\begin{array}{l}\text { Emit chemoattractants from } \\
\text { surface }\end{array}$ \\
\hline $\begin{array}{l}\text { Sense }(\text { Coord } \\
\mathrm{x}, \text { Coord } \mathrm{y})\end{array}$ & \begin{tabular}{|l} 
Sense chemoattractant con- \\
centration at surface sensors
\end{tabular} \\
\hline CalculateGradient() & $\begin{array}{l}\text { Calculate the chemical gradi- } \\
\text { ent using the values read at } 8 \\
\text { surface receptors }\end{array}$ \\
\hline FollowGradient() & $\begin{array}{l}\text { Move in the direction of the } \\
\text { chemoattractant gradient }\end{array}$ \\
\hline Random & $\begin{array}{l}\text { Take a random number of } \\
\text { steps in a randomly chosen } \\
\text { direction }\end{array}$ \\
\hline $\begin{array}{l}\text { Attach }(\text { Cell c1, Cell } \\
\mathrm{c} 2)\end{array}$ & $\begin{array}{l}\text { Attach to another cell after a } \\
\text { collision }\end{array}$ \\
\hline Proliferate(Cell c) & $\begin{array}{l}\text { Divide and create two new } \\
\text { cells }\end{array}$ \\
\hline Age & Increment internal clock \\
\hline $\operatorname{Die}($ Cell c) & \begin{tabular}{||l} 
Shut down receptors and stop \\
contributing/responding.
\end{tabular} \\
\hline
\end{tabular}

\subsection{Simulation Overview}

A cell's life cycle and behaviors are implemented as set of actions that are performed during each time step of a simulation. A single aggregation simulation is comprised of a series of these time steps. A cell's actions for each one of these time steps are outlined in Figure 9 and Table 2. Newly divided cells remain in a quiescent state for a user-defined period. These cells are not Active and cannot divide until they once again become Active. A quiescent cell can perform three types of actions. Immediately after cell division one of the new daughter cells separates from its sister cell. It moves with a user-defined velocity along the division axis for a user-defined period of time. Upon completion of this separation period the cell begins to emit a chemoattractant chemical and to respond to the chemoattractant gradient in its local environment. During the Quiescent phase there is some randomness in the cell's movement. These random motions vanish as the cell becomes Active. Based on the chemical fields produced by nearby cells a gradient is calculated and the cell/aggregate moves in the gradient direction in response to it. A cell is capable of attaching to other cells upon collision. A cell's age is incremented at each time step. If the cell is apoptotic, a check is then done to determine if the cell should die. If the state of the complete environment is to be saved for visualization purposes, the cell emits and stores its chemical field in the chemoattractant array. If in the Active stage, the cell determines if it is time to divide. If it divides, its and its daughter cell's age are set to zero, and they enter the Quiescent stage. The algorithms that implement the cell life cycle and its actions are detailed in Eyiyurekli et al. (2007).

\subsection{Experimental Model}

The PC12 cells used for experimental validation were cultured in T-flasks, in high glucose $(4.5 \mathrm{~g} / \mathrm{L})$ Dulbecco's modified Eagle's medium (DMEM), 


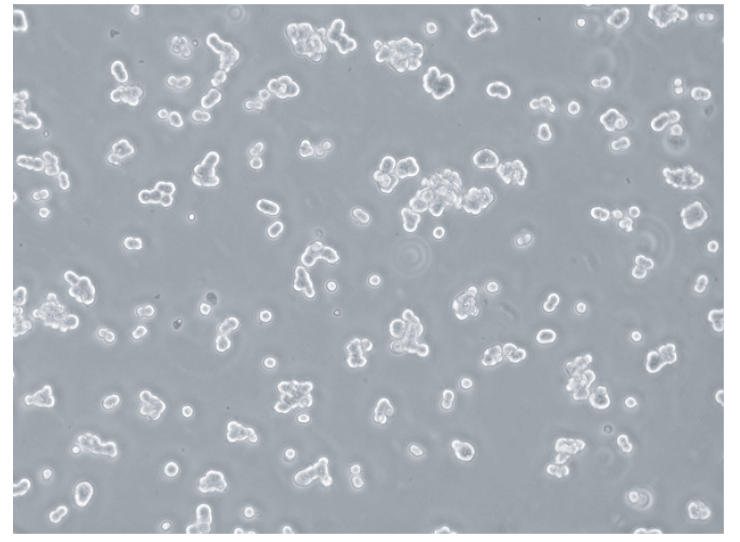

Experimental Data

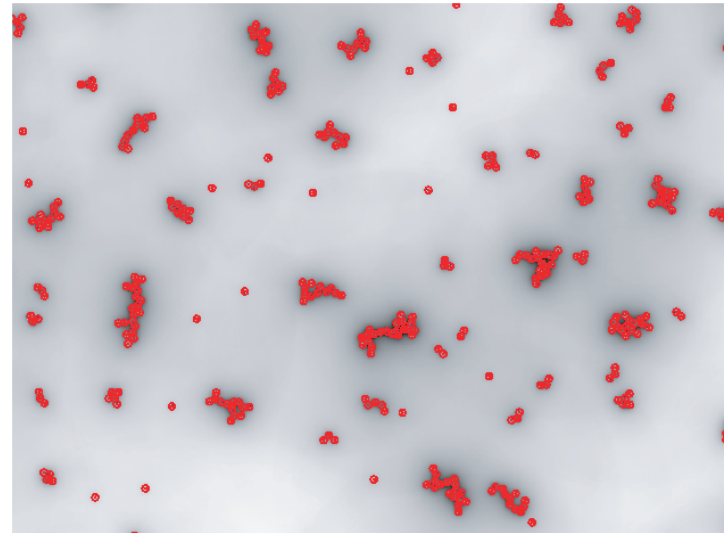

Simulation Results

Fig. 10. Comparison of live and simulated data. (left) Microscope image from in vitro experiment. (right) Visualization of simulated aggregation.

supplemented with $7.5 \%$ fetal bovine serum, $7.5 \%$ equine serum and L-Glutamine, in a $5 \% \mathrm{CO} 2$ incubator as previously described (Manley and Lelkes, 2006).

At time zero, PC12 cells were removed from a culture flask and re-suspended vigorously to break up aggregates. The cell suspension so obtained was seeded in a Petri dish through a $20 \mu \mathrm{m}$ cell strainer. This allows us to state with some degree of confidence that the sample, in the initial condition $(t=0)$ consisted mostly of single cells or aggregates consisting of at the most 2 or 3 cells. For validation experiments, the cells were seeded at a concentration of 50,000 cells per $\mathrm{ml}$ of culture medium. The cells were allowed to settle to the bottom of the Petri dish before microscopy. Some of these cells and small aggregates clumped together. In preliminary studies we determined that the number of aggregates depends on the initial seeding density (See Figure 10 (left)). Images were captured as soon as the cells settled, providing the initial data point $(\mathrm{t}=0)$. The Petri dishes were then placed back in the incubator and the cells were allowed to aggregate and proliferate for 24 hours before images were captured again $(\mathrm{t}=$ $24 \mathrm{hrs}$ ). For the studies described in this paper independent experiments were performed and the results averaged. The experimental data show a changing trend in favor of larger aggregates over time, which is what we attempted to simulate in our model. The images after 24 hours contain an increased number of cells and still do show some single cells rather than a population purely consisting of aggregates, which indicates active proliferation of cells in the culture.
The experimental data were gathered by manually analyzing twelve images that had been taken, with no spatial overlap, of different randomly located areas of the Petri dish. The images were acquired in a way to best represent the overall population of cells in the dish. The field of view captured in each image is $720 \mu \mathrm{m} \times 534.5 \mu \mathrm{m}$. Single PC12 cells are approximately $11 \mu \mathrm{m}$ in diameter with a standard deviation of about $4 \mu \mathrm{m}$. Manual analysis of the images consisted of counting the number of cells in each aggregate and tallying the aggregates with the same cell count. This produced aggregate size distributions for the 0th and 24th hours of the in vitro experiment, as listed in Table 4 and seen in Figures 12 and 13 (blue bars).

\section{Results}

An extensive number of simulations were performed with our computational model in order to determine which of its components are most critical for successfully simulating the aggregation behavior of PC12 cells. Our parametric studies initially used values taken from the literature for the parameters listed in Tables 1 and 3. We then systematically altered these parameter values, ran simulations, identified the most critical of the parameters, and the value ranges for each that would produce a close fit to the in vitro data. As we found a promising range for a particular parameter we conducted additional simulations at a higher sampling resolution within the range in order to pinpoint the optimal value, i.e. the parameter value that produced simulation 

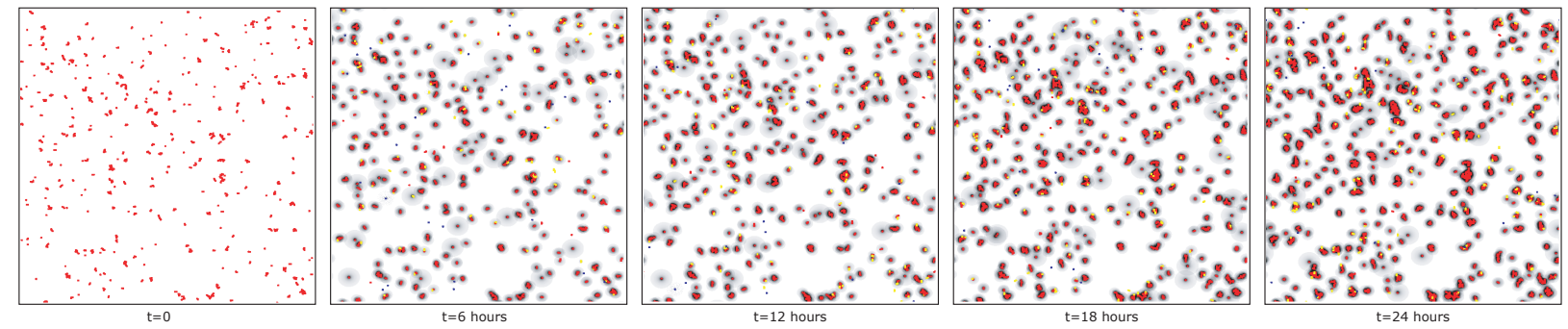

Fig. 11. Visualization of simulated cell aggregation. The state of the system (cell locations, cell life cycle state and chemoattractant concentration) can be stored and viewed at any time step.

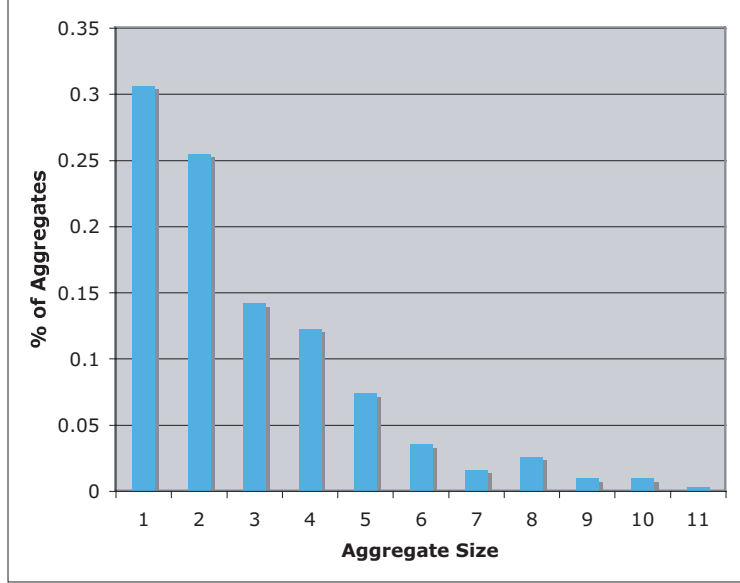

Fig. 12. Initial size distribution of aggregates gathered from the in vitro experiment. The $\mathrm{X}$ axis is the number of cells per aggregate. The $\mathrm{Y}$ axis is the percentage of aggregates of a particular cell count.

results best matching the in vitro results.

Initially, we studied the effect of chemoattractant emission up/down-regulation on aggregation. After numerous simulations, we found that we could only reproduce the in vitro results when emission was down-regulated as a function of cell attachments. We therefore used the values of $k_{2}$ defined by the blue line in Figure 4 when evaluating Equation 2 during all of our simulations. Additionally we explored a variety of probability profiles for $P_{S e p}$ that made it a function of the number of cell attachments. We investigated several functions that lowered the probability that a new daughter cell would separate after cell division as the number of cells attached to the parent cell increased. We found that this complicating feature did not improve our results. Keeping $P_{S e p}$ constant regardless of the number of cell attachments provided simulation results that best fit the in vitro results.

Starting with an initial cell/aggregate distribution derived from the $t=0$ in vitro experiment (see
Figure 12) we performed in silico aggregation experiments (simulations) equivalent to the 24 hour in vitro experiment described in the previous section. The simulations were performed in a 9,004,200 $\mu m^{2}(3225 \times 2792 \mu m)$ virtual Petri dish, with this area being equivalent to the total area of the in vitro experiment. At the end of each simulation the size distribution of the resulting aggregates is saved and compared with the aggregate size distribution derived from the in vitro experimental data. We also save the state of the simulation system and a representative image of the virtual Petri dish at regular intervals (see Figure 11).

The simulations were run on a 64-bit linux cluster running Dual-Opteron nodes with 1GB of RAM, and Gentoo 2.6. Individual execution times were primarily a function of the number of cells in the simulation. The average running time per simulation was approximately 30 minutes.

The first twenty entries of the 24th-hour size distribution from the simulated data are compared to the in vitro data using Earth Mover's Distance (EMD) (Rubner et al., 2000). EMD is a well-established method for computing the distance between two distributions. It is defined as the minimum amount of work required to transform one distribution into another, assuming that bin indices are equivalent to distance and the bin quantities are equivalent to mass. EMD provides a scalar measure that quantifies the similarity between the simulated and in vitro aggregate size distributions. While aggregates containing more than twenty cells were formed in both the in vitro and in silico experiments, most of the cells belong to the smaller aggregates. It was deemed more important to recreate the large-scale trend found in the smaller aggregates, rather than attempt to match the fewer and effectively randomly distributed larger aggregates.

The first set of parameters to be evaluated in our computational experiments was $\lambda$ (chemotactic re- 
Table 3

Critical parameters that affect cell aggregation. The range of parameter values utilized in the computational experiments. Parameter values that produced the averaged result with the best fit to the in vitro experimental results.

\begin{tabular}{|l||l|l|}
\hline Parameter & Range Tested & Value \\
\hline$P_{\text {Div }}^{0}$ & $0.001-0.15$ & 0.07 \\
\hline$\lambda$ & $0.01-0.1$ & 0.055 \\
\hline$T_{\text {Quiescent }}$ & 0 min-9 $\mathrm{hr}$ & $90 \mathrm{~min}$ \\
\hline$P_{\text {Sep }}$ & $0.0-1.0$ & 0.20 \\
\hline
\end{tabular}

sponse factor), $R_{M a x}$ (radius of influence), and $P_{D i v}^{0}$ We concluded that $R_{\text {Max }}$ should not be an independent parameter, but instead should be a function of $C_{0}, k_{1}$ and $k_{2}$, as defined by Equation 4 . Therefore it was removed from the tested variables in our parametric studies. After removing $R_{\text {Max }}$ from the variable test set $P_{D i v}^{0}$ values were restricted to only those producing an acceptable population doubling time and creating a size population similar to the in vitro experiment's. Another parameter that was modified and studied was $T_{\text {Quiescent }}$, the quiescent time period for newly created cells. The final parameter explored in these studies was $P_{S e p}$, the probability that two cells separate following cell division. The final series of computational experiments involved modifying the $P_{\text {Div }}^{0}, \lambda, T_{\text {Quiescent }}$, and $P_{\text {Sep }}$ parameters in order to find their values that would produce an optimal match between the in silico and in vitro aggregate distributions.

Each large series of simulations (consisting of $\sim 250$ runs) within the study utilized a range of values for a small subset of the model's parameters (See Table 3), keeping the majority of parameter values constant (See Table 1). For a particular set of parameter values five simulations were calculated, each using the same initial aggregate size distribution, but with different, randomly generated configurations/locations for the individual aggregates. After running five instances of a single 24-hour simulation a representative size distribution for the tested parameter set was produced by averaging together the distributions furnished by the individual simulations. By comparing the simulated results with the in vitro results using EMD and focusing our simulations on those parameter values producing the best match between the two, we manually searched the parameter space of the simulations to solve this non-linear optimization problem.

Table 3 presents the parameters identified as the most critical for reproducing PC12 cell aggregation

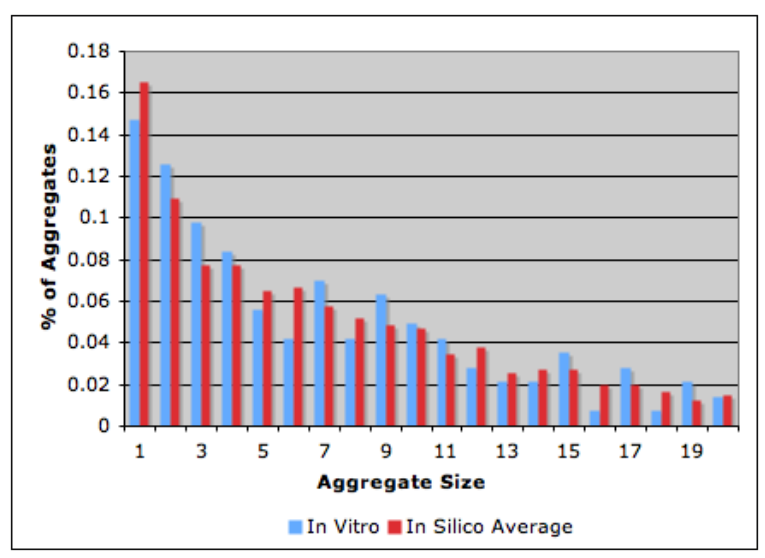

Fig. 13. Blue bars represent the aggregate size distribution observed in the in vitro experiment after 24 hours. The red bars are the size distribution produced by the average simulation with the best fit to the in vitro result.

behavior, with the parameter ranges tested during our study and optimal values found at the conclusion of our study. The parameter ranges were derived from the relevant literature. The range for $P_{D i v}^{0}$ gives doubling times from approximately two hours to several days. The optimal value of 0.07 produces a cell population doubling time of 12 hours, a typical value for PC12-like cells (Begg et al., 1985). This value of $P_{D i v}^{0}$ also produced approximately the same number of simulated cells in 24 hours that were present at the end of the in vitro experiment. The range of $\lambda$ values produces chemotaxisstimulated velocities from 1 to $10 \mu \mathrm{m} / \mathrm{min}$. The optimal value of 0.055 produces an average velocity of $5.5 \mu \mathrm{m} / \mathrm{min}$, a reasonable value for this type of cell (Song et al., 2006). The length of the quiescent time period was varied between 0 minutes and 9 hours (Lodish et al., 1995) and the simulations with the best-fitting result occurred when this period was 90 minutes. Finally, $P_{S e p}$ was varied through the full range of probability values from 0.0 and 1.0 , and a separation probability of 0.2 created the optimal result.

Figure 13 presents the distributions from best average simulation run and the in vitro experiment. The best single simulation result was contained in the series of in silico experiments that produced the optimal average. Its distribution is presented in Figure 14. Additionally the cell counts in these simulations also provided a good match with the in vitro experiment. Both the in vitro and in silico experiments started with 885 cells. After 24 hours the in vitro experiment contained 2764 cells. The best average simulation run had an average of 2915 cells 
Table 4

Size distributions for initial, 24-hour in vitro and equivalent simulated experiments. Values are the percentage of the total population existing in aggregates of various sizes.

\begin{tabular}{|l||l||l||l||l|}
\hline Aggregate Size & Initial $(0-\mathrm{hr})$ & $\begin{array}{l}\text { Experimental } \\
\text { hr) }\end{array}$ & $\begin{array}{l}\text { (24- } \\
\text { Best Average Simu- } \\
\text { lation }(24-\mathrm{hr})\end{array}$ & $\begin{array}{l}\text { Best Single Simula- } \\
\text { tion }(24-\mathrm{hr})\end{array}$ \\
\hline 1 & 0.3064 & 0.1468 & 0.1654 & 0.1503 \\
\hline 2 & 0.2548 & 0.1258 & 0.1097 & 0.1288 \\
\hline 3 & 0.1419 & 0.0979 & 0.0773 & 0.0889 \\
\hline 4 & 0.1225 & 0.0839 & 0.0773 & 0.0736 \\
\hline 5 & 0.0741 & 0.0559 & 0.0647 & 0.0736 \\
\hline 6 & 0.0354 & 0.0419 & 0.0665 & 0.0797 \\
\hline 7 & 0.0161 & 0.0699 & 0.0575 & 0.0521 \\
\hline 8 & 0.0258 & 0.0419 & 0.0521 & 0.0460 \\
\hline 9 & 0.0096 & 0.0629 & 0.0485 & 0.0368 \\
\hline 10 & 0.0096 & 0.0489 & 0.0467 & 0.0276 \\
\hline 11 & 0.0032 & 0.0419 & 0.0341 & 0.0460 \\
\hline 12 & 0 & 0.0279 & 0.0377 & 0.0398 \\
\hline 13 & 0 & 0.0209 & 0.0251 & 0.0214 \\
\hline 14 & 0 & 0.0209 & 0.0269 & 0.0245 \\
\hline 15 & 0 & 0.0349 & 0.0269 & 0.0276 \\
\hline 16 & 0 & 0.0069 & 0.0197 & 0.0030 \\
\hline 17 & 0 & 0.0279 & 0.0197 & 0.0276 \\
\hline 18 & 0 & 0.0069 & 0.016125 \\
\hline 19 & 0 & 0.0139 & 0.0122 \\
\hline 20 & 0 & & \\
\hline
\end{tabular}

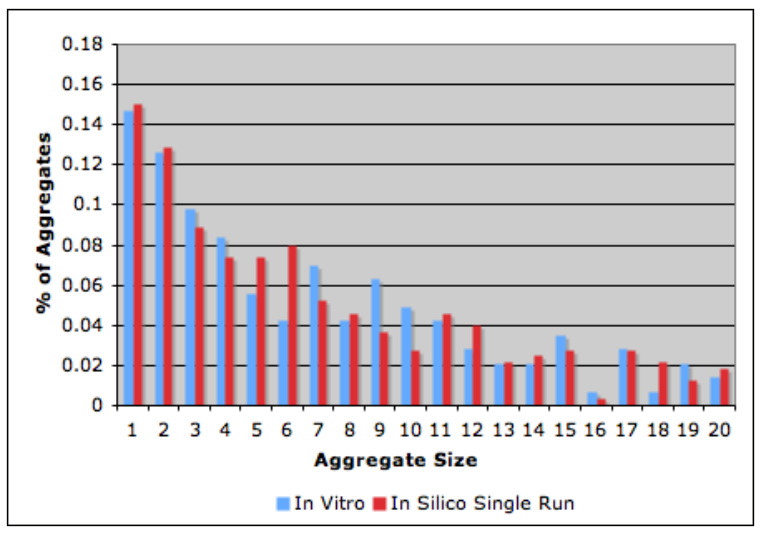

Fig. 14. Size distribution from the single simulation run (red bars) that produced the best fit to the in vitro result (blue bars).

after 24 simulation hours. The best individual simulation completed with 3007 cells. Table 4 contains the size distributions for the best average and best single simulations in comparison to the in vitro (after 24 hours) and initial size distributions. These results demonstrate that on average our computational model is capable of reproducing the largescale aggregation behavior, as represented by cell population size and aggregate size distributions, of PC12 cells.

\section{Parametric Studies}

Once the critical and inconsequential parameters were identified and parameter values optimized to produce the best fit to the in vitro results, parametric studies were conducted with the computational model to determine how each of the critical parameters affects the aggregation process. These studies highlight the usefulness of cell simulation, allowing us to perform computational experiments that may be difficult, time-consuming or impossible 


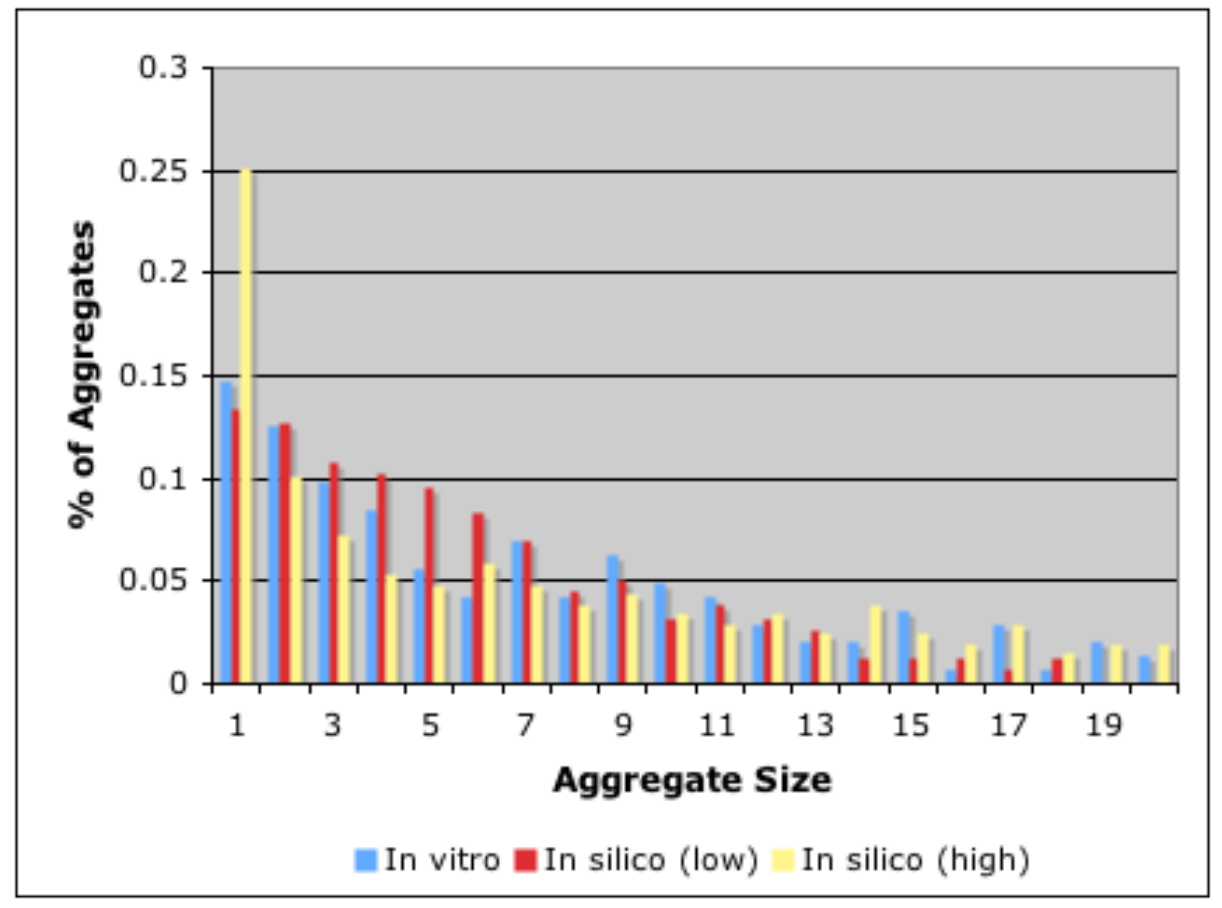

Fig. 15. Aggregation behavior as a function of proliferation rate. The cell proliferation rate directly affects the total number of cells. (red) Lower rates $\left(P_{D i v}^{0}=0.035\right)$ decreases the number of large aggregates and increases the number of smaller aggregates. (yellow) Higher rates $\left(P_{D i v}^{0}=0.14\right)$ dramatically increases the number of single cells, as well as overall population. The blue bars represent the aggregate size distribution from the in vitro experiment. This experiment began with 885 cell and contained 2764 cells after 24 hours. The red distribution contains 1773 cells and the yellow distribution contains 5916 cells after 24 hours.

to conduct in an experimental, in vitro setting. We see these types of computational studies leading to the design of specialized cells that can provide userspecified aggregation behavior.

In the parametric studies the values of $P_{D i v}^{0}$, $\lambda, T_{\text {Quiescent }}$ and $P_{\text {Sep }}$ were significantly modified from the optimal values, making them both larger and smaller. Additionally, up/down-regulation of chemoattractant emission was altered during our studies. The results from these parametric studies are described in detail below. All figures in this section compare the results of the parametric study simulations (red and yellow bars) with the aggregate size distribution from the in vitro experiment (blue bars).

$P_{\text {Div }}^{0}$ directly affects the size of the cell population since it determines how often cells proliferate. As the proliferation probability increases the environment becomes more crowded in a shorter period of time. For example, we started two sets of simulations with 885 cells, one with $P_{D i v}^{0}=0.035$ and other with $P_{D i v}^{0}=0.14$, keeping all other parameters constant. After 24 hours, the simulation with a lower proliferation rate had its population grow
100\%, completing with 1773 cells. The set with a higher proliferation rate had its population grow almost $560 \%$, completing with 5916 cells. The distributions from both of these simulations are shown in Figure 15. The $P_{D i v}^{0}$ parameter also has an effect on the size distribution of aggregates. As $P_{D i v}^{0}$ increases, the number of single cells increases. Outer layer cells in an aggregate are the ones that proliferate, and when an outer layer cell divides, the daughter cells sometimes stay together (see $P_{\text {Sep }}$ below) (Daniels et al., 2004). Thus, increased proliferation increases the number of larger aggregates as well as single cells. Lowering the proliferation probability lowers the number of cells, which lowers the rate of aggregation; thus producing a smaller number of larger aggregates.

$\lambda$ is the coefficient that affects a cell's speed in response to a chemical gradient. The speed of a cell affects the number and size of the aggregates formed. The faster the cells move in response to a chemical gradient the more likely they are to collide and attach before the apoptosis phase; thus lowering the number of dead cells at the end of the simulated 24 -hour period. We also observed that larger and 


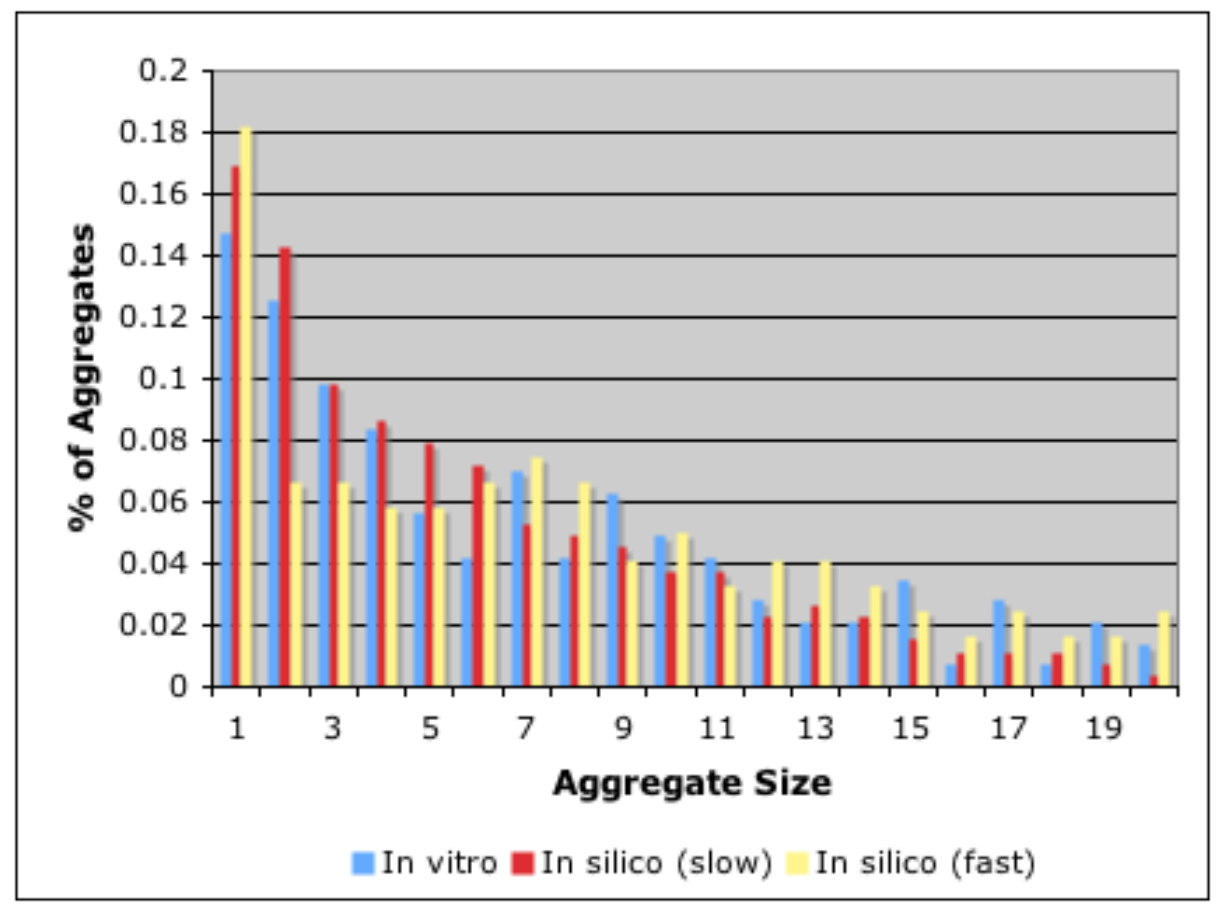

Fig. 16. Aggregation behavior as a function of chemoattractant gradient response. Slower cells $(\lambda=0.01)$ (red) form fewer and smaller aggregates while faster cells $(\lambda=0.1)$ (yellow) form larger aggregates, with an excess of single cells, after 24 hours. The blue bars represent the aggregate size distribution observed in the in vitro experiment after 24 hours.

more numerous aggregates form in a shorter period of time with an increase of the $\lambda$ parameter. In Figure 16 , red bars show the size distribution after 24 hours using slower cells $(\lambda=0.01)$, while the yellow bars show the same experiment with faster cells $(\lambda=0.1)$. As seen from the figure, slower cells tend to form smaller aggregates while faster cells form few smaller aggregates and more numerous larger aggregates after 24 hours. The spike in single cells for the faster cell population is caused by the lower overall number of aggregates produced by increased aggregation. With fewer aggregates the percentage of single cells in the population created via proliferation is enhanced. Changing the chemotactic response also affects proliferation. Since cells with more attachments proliferate less, as the aggregation rate is increased overall proliferation is reduced. Therefore the cell population of the slower cells is larger (3245) than the population of the faster cells (2607).

The effect of quiescent period length on aggregation behavior is shown in Figure 17. The $T_{\text {Quiescent }}$ parameter specifies how long a newly created cell remains inactive before being able to divide. Longer quiescent periods make it less likely that cells will form into aggregates, since chemotactic response is reduced during this period, leaving more single cells and fewer large aggregates. If this time period becomes too short, an aggressive proliferation behavior and increased aggregation is observed. In this case after 24 hours the number of smaller size aggregates decreases and more large ones are formed. The experiment with no quiescence resulted in 2955 cells and contained a 56 -cell aggregate. While a $T_{\text {Quiescent }}$ of $9 \mathrm{hrs}$ dropped the total cell count to 1905 with the largest aggregate containing 30 cells.

Figure 18 demonstrates how $P_{S e p}$ affects the aggregate size distribution in 24 hours. $P_{S e p}$ is the parameter that determines the probability that two cells detach and separate after cell division. If this probability is high then most cell divisions create single cells which then separate, resulting in a high percentage of single cells and fewer aggregates. If this probability is low then the number of single cells will not be as great and aggregates will grow bigger due to cell division on the outer layers. Setting the $P_{S e p}$ to zero prevents the production of new single cells. All aggregates then grow in size due to proliferation and aggregation, with the number of single cells and doublets going to zero. Since single cells have a higher probability of dividing, the value of $P_{S e p}$ that creates more single cells, i.e. a high value, also creates a larger final cell population. The exper- 


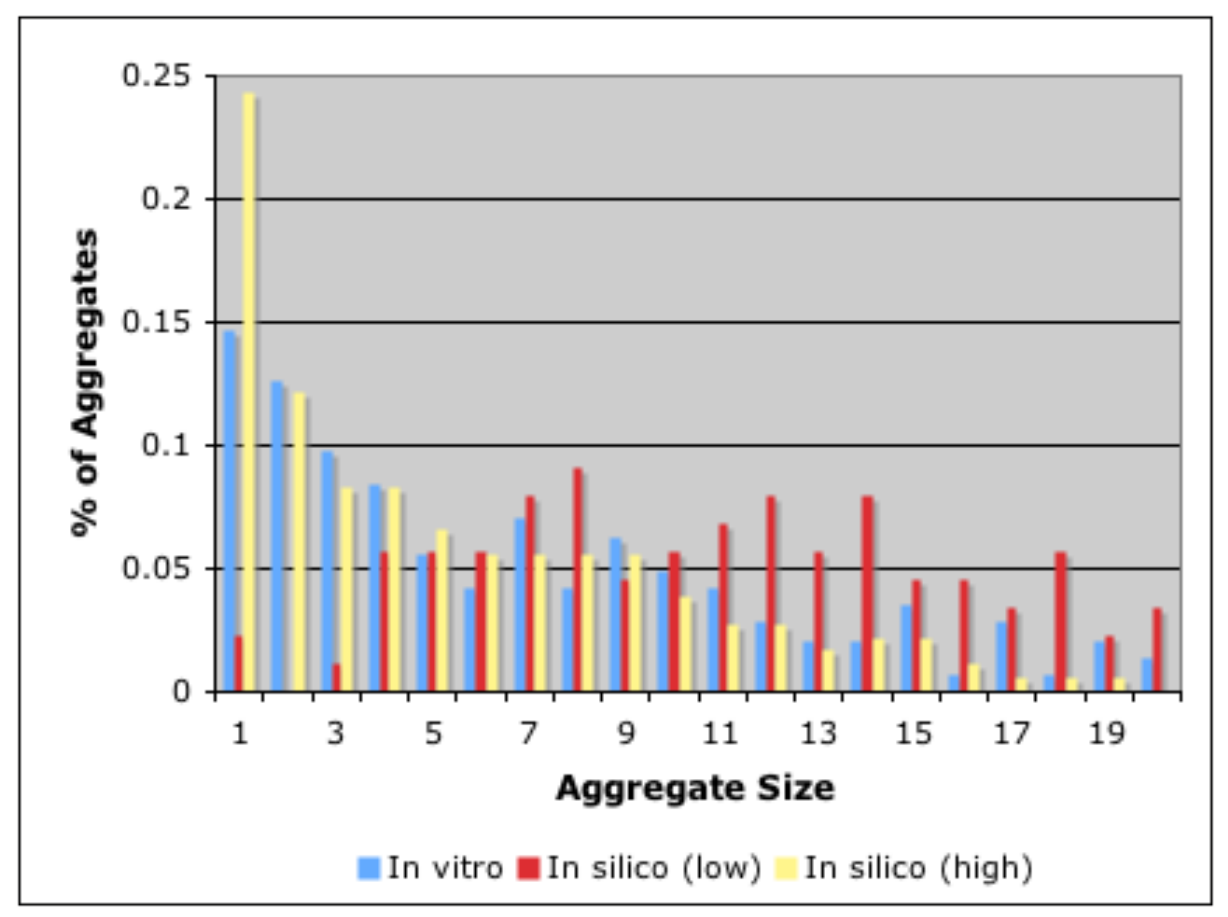

Fig. 17. Aggregation behavior as a function of quiescent period length. With no quiescent period $\left(T_{\text {Quiescent }}=0\right)$ cells aggregate quickly leaving few smaller aggregates (red). As $T_{\text {Quiescent }}$ increases fewer aggregates are formed (yellow).

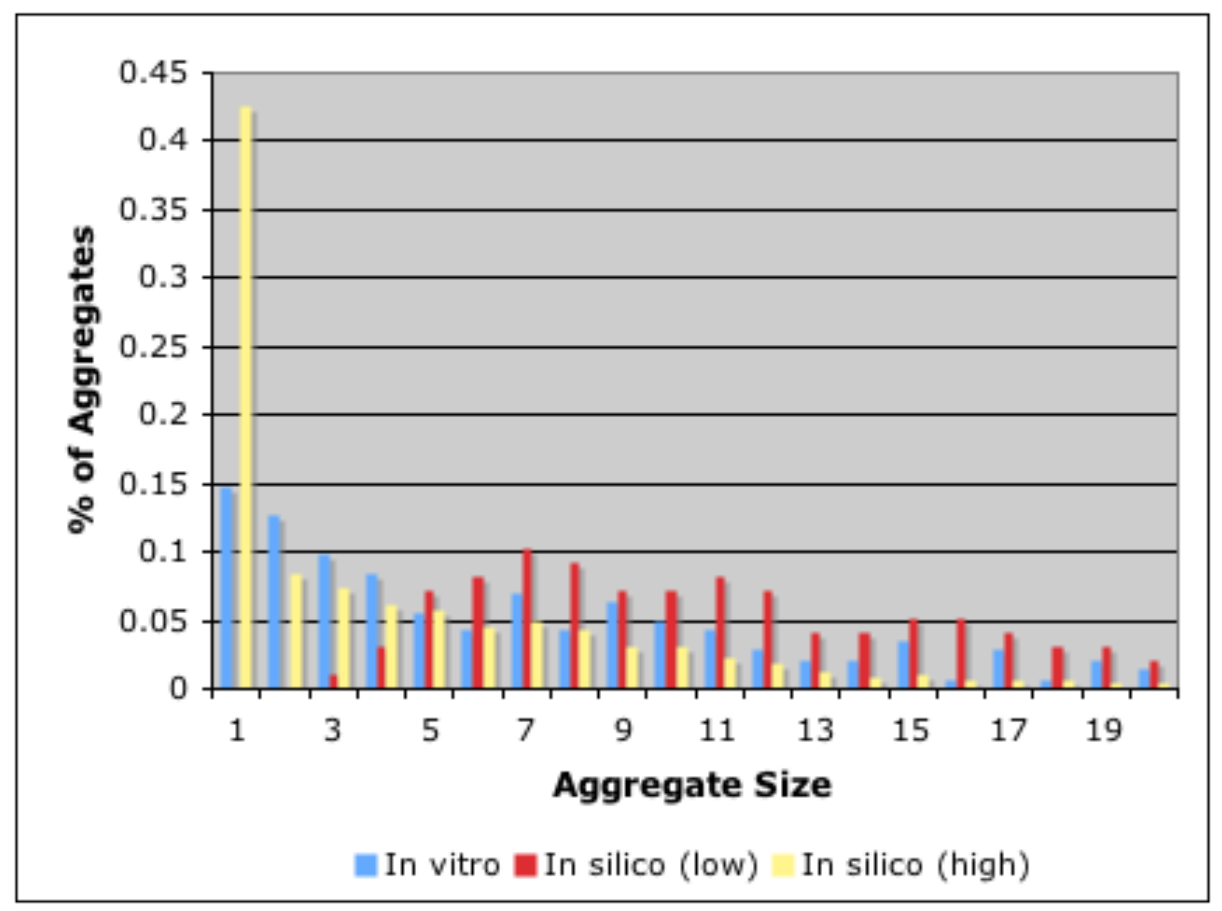

Fig. 18. Aggregation behavior as a function of $P_{S e p}$. (red) $P_{S e p}=0.0$. With no separation after proliferation, there are no singles and doublets, and more larger aggregates. (yellow) $P_{S e p}=1.0$. All cells separate after cell division, producing a high number of single cells and fewer large aggregates. Blue bars represent the aggregate size distribution observed in the in vitro experiment after 24 hours. 


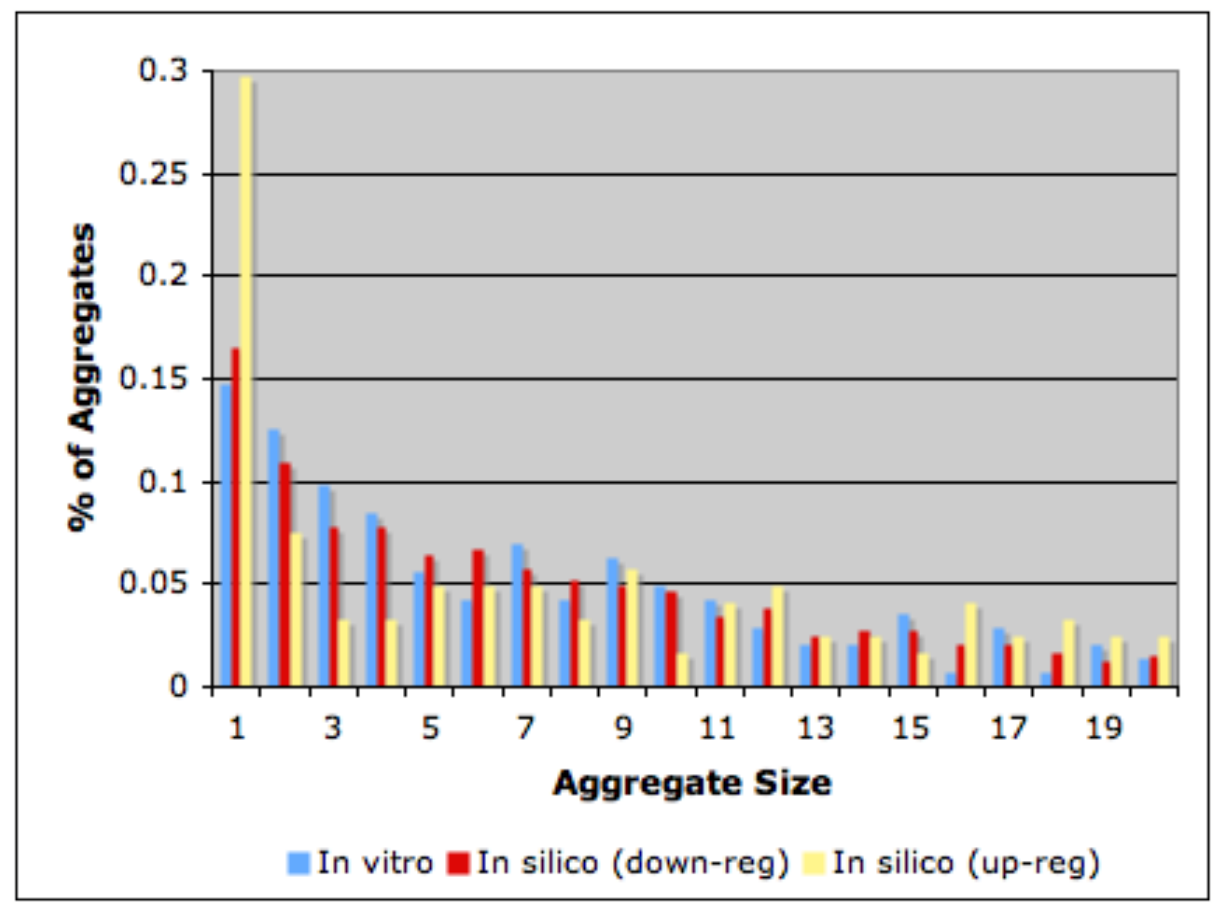

Fig. 19. Aggregation behavior as a function of up/down regulation of chemoattractant emission. (red) Emission is down-regulated as the number of attachments to a cell increases. This is the optimal result from Figure 13. (yellow) Emission is up-regulated as number of attachments increases. With the exception of the excessive number of single cells, larger aggregates are formed producing a more balanced distribution. Blue bars represent the aggregate size distribution observed in the in vitro experiment after 24 hours.

iment with $P_{\text {Sep }}$ equal to zero produced 2645 cells, and the experiment with $P_{S e p}$ equal to one produced 3877 cells.

The chemoattractant emission rate $\left(k_{2} * C_{0}\right)$ determines the effective concentration of chemoattractant at a cell's boundary, and therefore $R_{\text {Max }}$, the cell's range of influence. As the emission rate (and $R_{M a x}$ ) increases (via up-regulation based on the number of cell attachments and implemented with scale factor $k_{2}$ ) more cells and aggregates are attracted to each other over longer distances, and attachments occur more frequently; thus producing larger aggregates. Figure 19 demonstrates how changing the emission rates affects the size and number of the aggregates. The histogram with red bars is the same as Figure 13, and presents the distributions from best average simulation. The cells in this result down-regulate their chemoattractant emission as a function of the number of attached cells (blue line in Figure 4). We found that no up/down-regulation, up-regulation and a mixture of the two (red line, yellow line and green line in Figure 4) produced approximately the same aggregate size distribution, seen in Figure 19 (yellow bars). Here, emission is up-regulated as number of attachments increases, with a maximum $k_{2}$ value of four.

Whenever cells do not down-regulate chemoattractant emission, the aggregation process is accelerated, and the aggregate size distributions become flatter, with a spike in the single cells. Increased aggregation behavior yields a lower overall number of aggregates that tend to be large in size, The optimized, down-regulated simulation shown in red bars contains 556 aggregates, 92 of which are single cells, and with the largest aggregate containing 50 cells. The up-regulated simulation contains 121 aggregates, of which 36 are singles cells, and with the largest aggregate containing 90 cells. As with the other parameters, increasing the rate of aggregation lessens proliferation. The lessening effect was not as significant in this particular study, with the downregulated simulation producing 2915 cells and the up-regulated simulation producing 2528 cells.

The spike in the percentage of single cells seen in Figure 19 (yellow bars) should not be interpreted as an excessive number of single cells, but rather is caused by a decrease in the production of double, triple and quadruple-cell aggregates. As single cells 
are created via proliferation they are more likely to be attracted to larger aggregates, rather then to each. This process increases the size of already large aggregates, and lowers the number and percentage of the smaller aggregates. A similar phenomenon was observed when increasing the aggregation rate by increasing $\lambda$, as seen in Figure 16.

\section{Conclusion}

We have presented a 2-D chemotaxis-based computational model that successfully captures many of the cell behaviors that play important roles in cell aggregation. A virtual cell in our model is designed as an independent, discrete unit with a collection of parameters and actions. Each cell is defined by its location, size, chemoattractant emission and response rates, age, life cycle stage, proliferation rate and number of attached cells. All cells are capable of emitting and sensing chemoattractant chemical, moving, attaching to other cells, dividing, aging and dying.

We validated and fine-tuned our model by comparing simulated 24-hour aggregation experiments with data derived from in vitro experiments using PC12 cells. Quantitative comparisons of the aggregate size distributions from the two experiments were produced using the Earth Mover's Distance (EMD) metric. We compared the two size distributions produced after 24 hours of in vitro cell aggregation and the equivalent computer simulated process. Iteratively modifying the model's parameter values and measuring the difference between the in silico and in vitro results allowed us to determine the optimal values to produce simulated aggregation outcomes closely matched to the PC12 experiments. Results shown in Section 4 confirm the ability of our model to recreate large-scale aggregation behaviors seen in live cell experiments.

Through our simulation studies we were able to identify important factors affecting cell aggregation such as the cell's proliferation rate, response rate to chemoattractant gradient, length of the quiescent stage after cell division and up/down-regulation of chemoattractant emission based on the number of attached cells.

\section{Acknowledgements}

This research was funded by National Science Foundation grant CCF-0636323 and NASA grants
NCC9-130 and NAG97-HEDS-02.

\section{References}

Ascencio, S. F., Meana, H. P., Miyatake, M. N., 2001. Two and three dimensional computer simulation of cancer growth. Proc. XXI Int. Conference of the Chilean Computer Science Society, 7379 .

Bagchi, P., Johnson, P. C., Popel, A. S., 2005. Computational fluid dynamic simulation of aggregation of deformable cells in a shear flow. Transactions of the ASME 127, 1070.

Begg, A. C., McNally, N. J., Shrieve, D. C., Karcher, H., 1985. A method to measure the duration of DNA synthesis and the potential doubling time from a single sample. Cytometry 6, 620-626.

Bio-SPICE, 2008. Bio-SPICE: A simulation program for intra- and inter-cell evaluation.

URL http://biospice.sourceforge.net

Castor, L. N., 1972. Contact inhibitions of cell division and cell movement. Journal of Investigative Dermatology 59, 27-32.

Chaturvedi, R., Huang, C., Kazmierczak, B., Schneider, T., Izaguirre, J. A., Newman, S. A., Glazier, J. A., Alber, M., 2005. On multiscale approaches to three-dimensional modeling of morphogenesis. Journal of the Royal Society 2, 237253.

Christopher, R., Dhiman, A., Fox, J., Gendelman, R., Haberitcher, T., Kagle, D., Spizza, G., Khalil, I. G., Hill, C., 2004. Data-driven computer simulation of human cancer cell. Annals of NY Academy of Science 1020, 132-153.

Cickovski, T., et al., 2005. A framework for three-dimensional simulation of morphogenesis. IEEE/ACM Transactions on Computational Biology and Bioinformatics 2, 273-288.

Crank, J., 1975. The Mathematics of Diffusion, 2nd Edition. Oxford University Press, Oxford.

Daniels, M. J., Wang, Y., Lee, M., Venkitaraman, A. R., 2004. Abnormal cytokinesis in cells deficient in the breast cancer susceptibility protein BRCA2. Science 306, 876-879.

Dormann, S., Deutsch, A., 2002. Modeling of selforganized avascular tumor growth with a hybrid cellular automaton. In Silico Biology 2 (3), 393406.

Emonet, T., Macal, C. M., North, M. J., Wickersham, C. E., Cluzell, P., 2005. AgentCell: a digital single-cell assay for bacterial chemotaxis. Bioin- 
formatics 21 (11), 2714-2721.

Eyiyurekli, M., 2006. A computational model of chemotaxis-based cell aggregation. Master's thesis, Drexel University, Philadelphia, PA.

Eyiyurekli, M., Lelkes, P., Breen, D., 2007. A computational system for investigating chemotaxisbased cell aggregation. In: Proc. European Conference on Artificial Life. pp. 1034-1049.

Farrell, B. E., Daniele, R. P., Lauffenburger, D. A., 1990. Quantitative relationships between singlecell and cell-population model parameters for chemosensory migration responses of alveolar macrophages to C5a. Cell Motility and the $\mathrm{Cy}-$ toskeleton 16, 279-293.

Fleischer, K. W., 1995. Multiple-mechanism developmental model for defining self-organizing geometric structures. Ph.D. thesis, California Institute of Technology.

Fleischer, K. W., 1996. Investigations with a multicellular developmental model. Artificial Life V, 229-236.

Fleischer, K. W., Barr, A. H., 1994. A simulation testbed for the study of multicellular development: the multiple mechanisms of morphogenesis. Artificial Life III, 389-408.

Frisch, S. M., Francis, H., 1994. Disruption of epithelial cell-matrix interactions induces apoptosis. Journal of Cell Biology 124 (4), 619-626.

Glazier, J. A., Graner, F., 1993. Simulation of the differential adhesion driven rearrangement of biological cells. Physical Review E 47, 2128-2154.

Goldberg, D. E., 1989. Genetic Algorithms in Search, Optimization, and Machine Learning. Addison-Wesley.

Graner, F., Glazier, J. A., 1992. Simulation of biological cell sorting using a two-dimensional extended Potts model. Physical Review Letters 69, 2013-2016.

Hirata, Y., Adachi, K., Kiuchi, K., 1998. Activation of JNK pathway and induction of apoptosis by manganese in PC12 cells. Journal of Neurochemistry $71,1607-1615$.

Hogeweg, P., 2000. Evolving mechanisms of morphogenesis: on the interplay between differential adhesion and cell differentiation. Journal of Theoretical Biology 203, 317-333.

Hogeweg, P., 2002. Computing an organism: on the interface between informatic and dynamic processes. Biosystems 64, 97-109.

Huttenlocher, A., Lakonishok, M., Kinder, M., Wu, S., Truong, T., Knudsen, K. A., Horwitz, A. F., 1998. Integrin and cadherin synergy regulates contact inhibition of migration and motile activity. The Journal of Cell Biology 141, 515-526.

Ilachinski, A., 2001. Cellular Automata : A Discrete Universe. World Scientific, Singapore.

Izaguirre, J. A., Chaturvedi, R., Huang, C., Cickovski, T., Coffland, J., Thomas, G., Forgacs, G., Alber, M., Newman, S., Glazier, J. A., 2004. CompuCell, a multi-model framework for simulations of morphogenesis. Bioinformatics 20, 1129-1137.

Jabbarzadeh, E., Abrams, C. F., 2005. Chemotaxis and random motility in unsteady chemoattractant fields: A computational study. Journal of Theoretical Biology 235, 221-232.

Jiang, Y., Pjesivac-Grbovic, J., Cantrell, C., Freyer, J., 2005. A multiscale model for avascular tumor growth. Biophysical Journal 89, 3884-3894.

Kobayashi, S. D., Voyich, J. M., Braughton, K. R., DeLeo, F. R., 2003. Down-regulation of proinammatory capacity during apoptosis in human polymorphonuclear leukocytes. The Journal of Immunology 170, 3357-3368.

Kumar, S., 2004. Investigating computational models of development for the construction of shape and form. Ph.D. thesis, University College London.

Lodish, H. F., Baltimore, D., Berk, A., Zipursky, S. L., Matsudaira, P., Darnell, J. E., 1995. Molecular Cell Biology, 3rd Edition. W. H. Freeman, New York, NY, USA.

Longo, D., Peirce, S. M., Skalak, T. C., Davidson, L., Marsden, M., Dzamba, B., Simone, D. W. D., 2004. Multicellular computer simulation of morphogenesis: blastocoel roof thinning and matrix assembly in Xenopus laevis. Developmental Biology $271,210-222$.

Manley, P., Lelkes, P. I., 2006. A novel real-time system to monitor cell aggregation and trajectories in rotating wall vessel bioreactors. Journal of Biotechnology 125 (3), 416-424.

Merks, R. M. H., Glazier, J. A., 2005. A cell-centered approach to developmental biology. Physica A 352, 113-130.

N'Dri, N. A., Shyy, W., Tran-Son-Tay, 2003. Computational modeling of cell adhesion and movement using a continuum-kinetics approach. Biophysical Journal 85, 2273-2286.

Newman, S., et al., 2008. Multiscale models for vertebrate limb development. Current Topics in Developmental Biology 81, 311-340.

Palsson, E., Othmer, H., 2000. A model for individual and collective cell movement in dictyostelium discoideum. Proceedings of the $\mathrm{Na}$ - 
tional Academy of Science USA 97, 10448-10453. Patel, A. A., Gawlinski, E. T., Lemieux, S. K., Gatenby, R. A., 2001. A cellular automation model of early tumor growth and invasion: the effects of native tissue vascularity and increase in anaerobic tissue metabolism. Journal of Theoretical Biology 213, 315-331.

Rubner, Y., Tomasi, C., Guibas, L., 2000. The earth mover's distance as a metric for image retrieval. International Journal of Computer Vision 40 (2), 99-121.

Savinell, J. M., Lee, G. M., Palsson, B. O., Arbor, A., 1989. On the orders of magnitude of epigenic dynamics and monoclonal antibody production. Bioprocess Engineering 4, 231-234.

Schutz, L., Mora, P., 1968. The need for direct cell contact in 'contact' inhibition of cell division in culture. Journal of Cellular Physiology 71 (1), 16.

Serini, G., et al., 2003. Modeling the early stages of vacular network assembly. The EMBO Journal 22 (8), 1771-1779.

Slepchenko, B. M., Schaff, J. C., Macara, I., Loew, L. M., 2003. Quantitative cell biology with the virtual cell. Trends in Cell Biology 13 (11), 570576.

Song, H., Jain, S. K., Enmon, R. M., O'Connor, K. C., 2004. Restructuring dynamics of DU 145 and LNCaP prostate cancer spheroids. In Vitro Cellular and Developmental Biology-Animal 40, 262-267.

Song, L., Nadkarni, S. M., Bodeker, H. U., Beta, C., Bae, A., Franck, C., Rappel, W. J., Loomis, W. F., Bodenschatz, E., 2006. Dictyostelium discoideum chemotaxis: Threshold for directed motion. European Journal of Cell Biology 85, 981-989.

Sozinova, O., Jiang, Y., Kaiser, D., Alber, M., 2006. Three-dimensional model of fruiting body formation. Proceedings of the National Academy of Science USA 103, 17255-17259.

Takahashi, K., Kaizu, K., Hu, B., Tomita, M., 2004. A multi-algorithm, multi-timescale method for cell simulation. Bioinformatics 20 (4), 538-546.

Thomas, L. A., Yamada, K. M., 1992. Contact stimulation of cell migration. Journal of Cell Science 103, 1211-1214.

Tomita, M., et al., 1999. E-Cell: Software environment for whole cell simulation. Bioinformatics 15 , $72-84$.

Tozeren, A., Coward, C. W., Petushi, S. P., 2005. Origins and evolution of cell phenotypes in breast tumors. Journal of Theoretical Biology 233, 43-
54.

Wall, D., Wu, S. S., Kaiser, D., 1998. Contact stimulation of Tgl and type IV Pili in Myxococcus xanthus. Journal of Bacteriology 180, 759-761.

Watanabe, O., Torda, M., Meldolesi, J., 1983. The effect of $\alpha$-Latrotoxin on the neurosecretory PC12 cell line: Electron microscopy and cytotoxicity studies. Neuroscience 10 (3), 1011-1024.

Wolfram, S., 1984. Cellular automata as models of complexity. Nature 311, 419-424. 